\title{
Bifurcation Analysis and Different Kinds of Exact Travelling Wave Solutions of a Generalized Two-Component Hunter-Saxton System
}

\author{
Qing Meng ${ }^{1}$ and Bin $\mathrm{He}^{2}$ \\ ${ }^{1}$ Department of Physics, Honghe University, Mengzi, Yunnan 661100, China \\ ${ }^{2}$ College of Mathematics, Honghe University, Mengzi, Yunnan 661100, China \\ Correspondence should be addressed to Bin He; hebinmtc@163.com
}

Received 22 March 2014; Accepted 27 May 2014; Published 18 June 2014

Academic Editor: Hagen Neidhardt

Copyright (C) 2014 Q. Meng and B. He. This is an open access article distributed under the Creative Commons Attribution License, which permits unrestricted use, distribution, and reproduction in any medium, provided the original work is properly cited.

This paper focuses on a generalized two-component Hunter-Saxton system. From a dynamic point of view, the existence of different kinds of periodic wave, solitary wave, and blow-up wave is proved and the sufficient conditions to guarantee the existence of the above solutions in different regions of the parametric space are given. Also, some exact parametric representations of the travelling waves are presented.

\section{Introduction}

The Hunter-Saxton equation,

$$
u_{t x x}+2 u_{x} u_{x x}+u u_{x x x}=0
$$

was first introduced by Hunter and Saxton [1] as a model of the dynamics of a nematic liquid crystal. Geometrically, the HS equation (1) describes geodesic flow associated with the right-invariant metrics on a homogeneous space [2]. It is a particular case of the Euler-Poincaré equation on the diffeomorphisms in one spatial dimension [3]. Its integrability was proved by Hunter and Zheng [4].

The two-component Hunter-Saxton system is as follows:

$$
\begin{gathered}
u_{t x x}+2 u_{x} u_{x x}+u u_{x x x}-\rho \rho_{x}=0, \\
\rho_{t}+(\rho u)_{x}=0,
\end{gathered}
$$

which is a generalization of the Hunter-Saxton equation, and was proposed by Wunsch [5] in a periodic setting. It is a particular case of the Gurevich-Zybin system [6] pertaining to nonlinear one-dimensional dynamics of dark matter as well as nonlinear ion-acoustic waves [7]. The two-component system in a periodic setting has received some attention lately $[8,9]$.
Very recently, the generalized two-component HunterSaxton system

$$
\begin{gathered}
u_{t x x}+2 \sigma u_{x} u_{x x}+\sigma u u_{x x x}-\rho \rho_{x}+A u_{x}=0, \\
\rho_{t}+(\rho u)_{x}=0, \quad \sigma \in R, \quad A \geq 0,
\end{gathered}
$$

was studied [10-12]. Moon and Liu [10] studied the wavebreaking phenomena and global existence for (3). Moon [11] determined the existence of solitary wave solutions for $\sigma=0$ and classified the solitary waves for $\sigma \neq 0$. The existence of peaked solitary waves for $\sigma>1$ was shown. The wavebreaking criterion and local well-posedness were studied in [12].

In this paper, we investigated the following generalized two-component Hunter-Saxton system:

$$
\begin{gathered}
u_{t x x}+2 u_{x} u_{x x}+u u_{x x x}-\rho \rho_{x}+A u_{x}=0, \\
\rho_{t}+(\rho u)_{x}=0, \quad A \in R .
\end{gathered}
$$

Obviously, system (4) becomes system (2) when $A=0$. We will prove the existence of different kinds of travelling wave of (4) and give some new exact travelling solutions using the approach of dynamical system [13-17]. 
Using the following independent variable transformation:

$$
u(x, t)=u(\xi), \quad u(x, t)=\rho(\xi), \quad \xi=x-c t,
$$

where $c(c \neq 0)$ is the wave speed, and substituting (5) into (4), we obtain

$$
\begin{gathered}
-c u^{\prime \prime \prime}+2 u^{\prime} u^{\prime \prime}+u u^{\prime \prime \prime}-\rho \rho^{\prime}+A u^{\prime}=0, \\
-c \rho^{\prime}+(\rho u)^{\prime}=0,
\end{gathered}
$$

where "' " is the derivative with respect to $\xi$.

Integrating equations of (6) once with respect to $\xi$, respectively, and setting the integral constants that are both $-2 c^{2}$, we have

$$
\begin{gathered}
-c u^{\prime \prime}+\frac{1}{2}\left(u^{\prime}\right)^{2}+u u^{\prime \prime}-\frac{1}{2} \rho^{2}+A u=-2 c^{2}, \\
-c \rho+(\rho u)=-2 c^{2} .
\end{gathered}
$$

The second equation of (7) becomes

$$
\rho=-\frac{2 c^{2}}{c-u} .
$$

Substituting (8) into the first equation of (7) yields

$$
\begin{aligned}
& u^{\prime \prime} \\
& =\frac{u\left(A u^{2}-2 c(A-c) u+c^{2}(A-4 c)\right)+(1 / 2)(c-u)^{2}\left(u^{\prime}\right)^{2}}{(c-u)^{3}} .
\end{aligned}
$$

Letting $y=d u / d \xi$, we get the following planar dynamical system:

$$
\begin{aligned}
& \frac{d u}{d \xi}=y, \\
& \frac{d y}{d \xi} \\
& =\frac{u\left(A u^{2}-2 c(A-c) u+c^{2}(A-4 c)\right)+(1 / 2)(c-u)^{2} y^{2}}{(c-u)^{3}} .
\end{aligned}
$$

The rest of this paper is organized as follows. In Section 2, we discuss the bifurcation sets and phase portraits of system (10), where explicit parametric conditions will be derived. In Section 3, we give all possible exact periodic wave, solitary wave, and blow-up wave solutions of system (4). A short conclusion will be given in Section 4 .

\section{Bifurcation Sets and Phase Portraits of System (10)}

Using the transformation $d \xi=(c-u)^{3} d \tau$, it carries (10) into the Hamiltonian system:

$$
\begin{gathered}
\frac{d u}{d \tau}=(c-u)^{3} y \\
\frac{d y}{d \tau}=u\left(A u^{2}-2 c(A-c) u+c^{2}(A-4 c)\right)+\frac{1}{2}(c-u)^{2} y^{2}
\end{gathered}
$$

with the following first integral:

$$
\begin{aligned}
H(u, y) & =\frac{(c-u)^{2} y^{2}+A u^{3}-c(A-4 c) u^{2}-4 c^{3} u+4 c^{4}}{c-u} \\
& =h
\end{aligned}
$$

For a fixed $h$, the level curve $H(\phi, y)=h$ defined by (12) determines a set of invariant curves of system (11) which contains different branches of curves. As $h$ is varied, it defines different families of orbits of (11) with different dynamical behaviors.

Write $\Delta=c(2 A+c)$. Clearly, when $A \neq 0$ and $\Delta>0$, system (11) has three equilibrium points at $(0,0),\left(u_{1}, 0\right)$, and $\left(u_{2}, 0\right)$ in $u$-axis, where $u_{1,2}=c((A-c) \pm \sqrt{\Delta}) / A$. When $A \neq 0$ and $\Delta=0$, system (11) has two equilibrium points at $(0,0)$ and $(c(A-c) / A, 0)$ in $u$-axis. When $A=0$, system (11) has only one equilibrium point at $(0,0)$ in $u$-axis. There is no any equilibrium point of system (11) in line $u=c$.

Let $M\left(\phi_{e}, y_{e}\right)$ be the coefficient matrix of the linearized system of (11) at equilibrium point $\left(\phi_{e}, y_{e}\right)$, then we have Trace $M\left(\phi_{e}, 0\right)=0$ and

$$
\begin{aligned}
J(0,0) & =\operatorname{det} M(0,0)=-c^{5}(A-4 c), \\
J\left(u_{1}, 0\right) & =\operatorname{det} M\left(u_{1}, 0\right) \\
& =\frac{2 c^{5}(-c+\sqrt{\Delta})^{3}(c(2 A+c)+(A-c) \sqrt{\Delta})}{A^{4}}, \\
J\left(u_{2}, 0\right) & =\operatorname{det} M\left(u_{2}, 0\right) \\
& =\frac{2 c^{5}(c+\sqrt{\Delta})^{3}(-c(2 A+c)+(A-c) \sqrt{\Delta})}{A^{4}} .
\end{aligned}
$$

For an equilibrium point $\left(\phi_{e}, y_{e}\right)$ of a planar integrable system, we know that $\left(\phi_{e}, y_{e}\right)$ is a saddle point if $J\left(\phi_{e}, y_{e}\right)<0$, a center point if $J\left(\phi_{e}, y_{e}\right)>0$ and Trace $M\left(\phi_{e}, y_{e}\right)=0$, and a cusp if $J\left(\phi_{e}, y_{e}\right)=0$ and the Poincaré index of $\left(\phi_{e}, y_{e}\right)$ is zero.

Since both systems (10) and (11) have the same first integral (12), then the two systems above have the same topological phase portraits. Therefore we can obtain the bifurcation sets and phase portraits of system (10) from that of system (11).

By using the properties of equilibrium points and bifurcation method of dynamical systems, we can show that bifurcation sets and phase portraits of system (10) are drawn in Figure 1. 


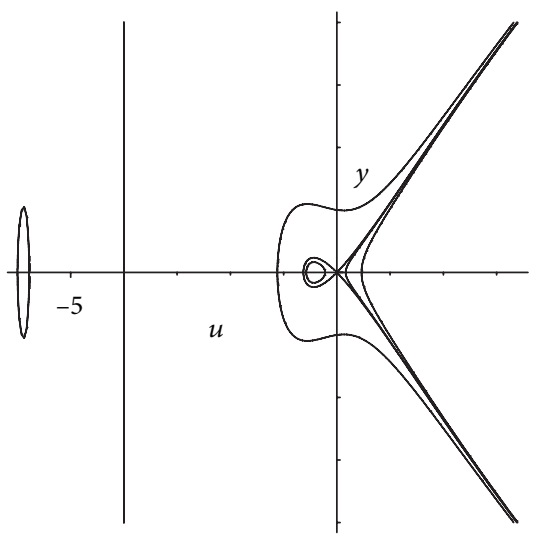

(a) $c<0, A<4 c$

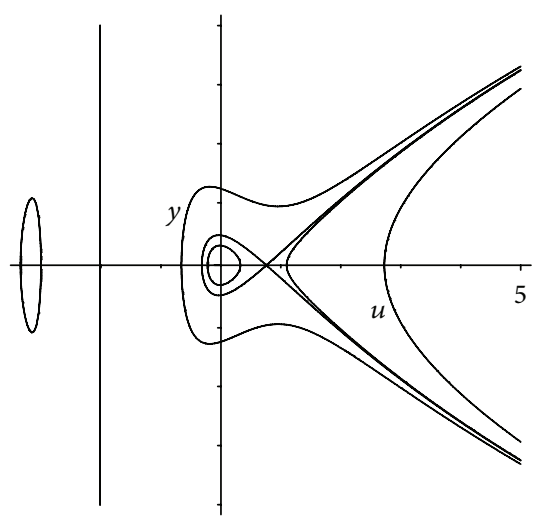

(c) $c<0,4 c<A<0$

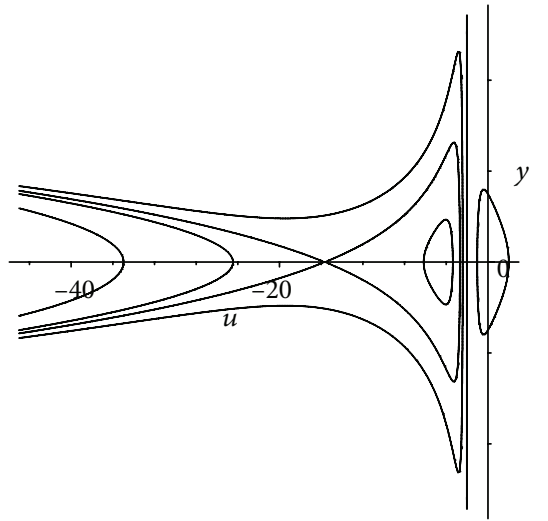

(e) $c<0,0<A<-(1 / 2) c$

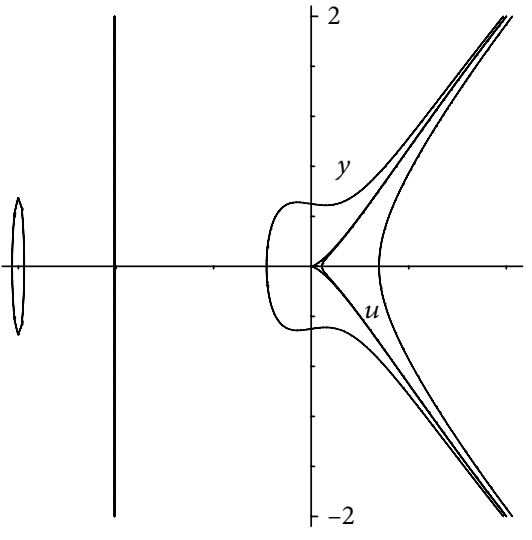

(b) $c<0, A=4 c$

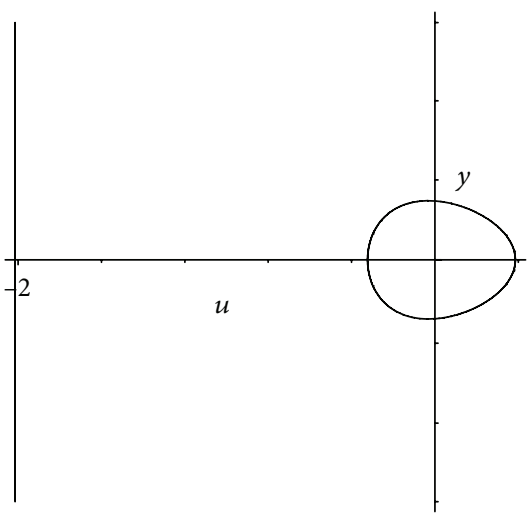

(d) $c<0, A=0$

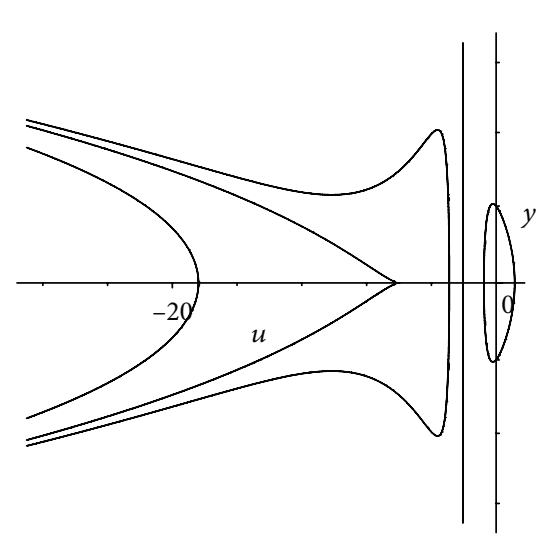

(f) $c<0, A=-(1 / 2) c$

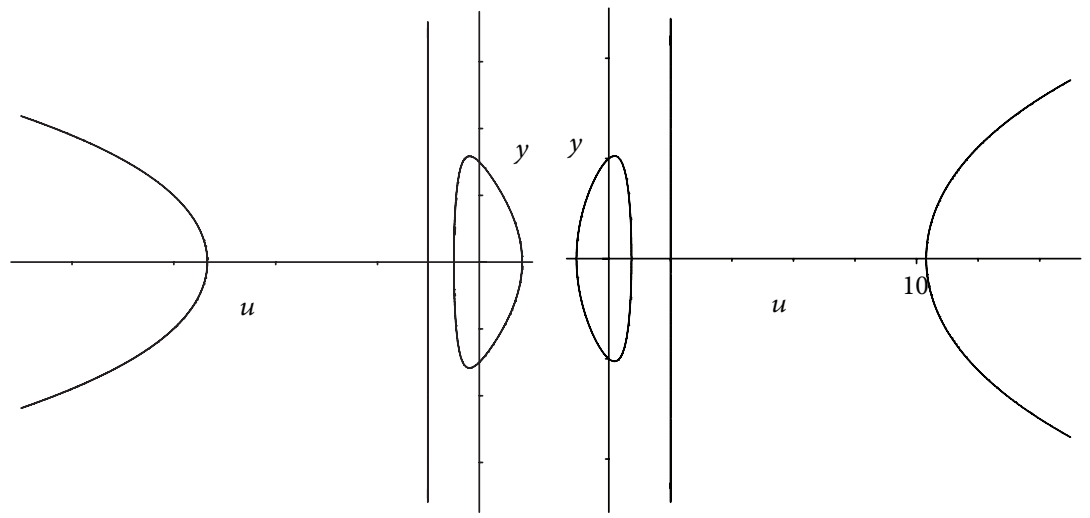

(g) $c<0, A>-(1 / 2) c$

(h) $c>0, A<-(1 / 2) c$

FIgURE 1: Continued. 


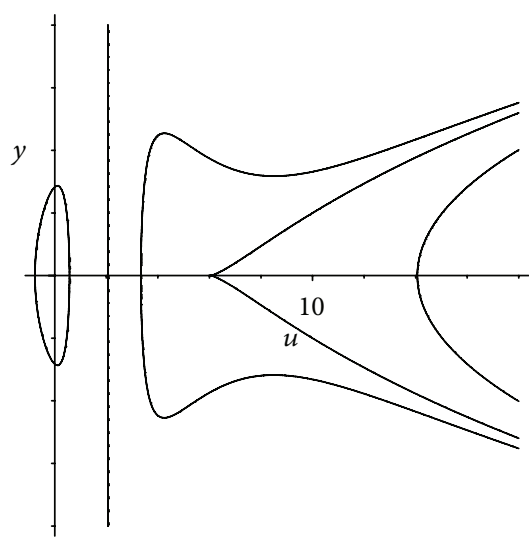

(i) $c>0, A=-(1 / 2) c$

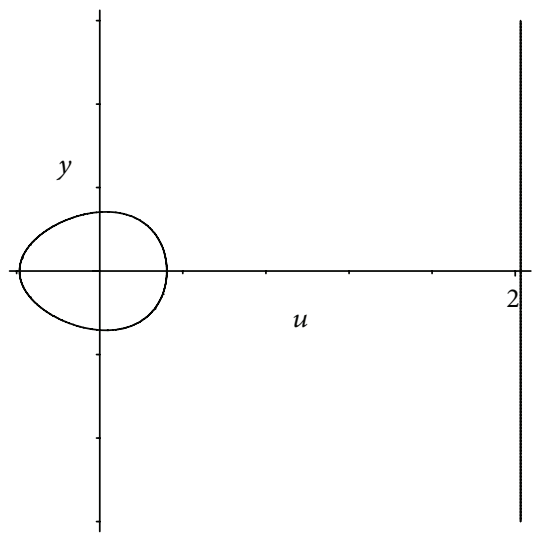

(k) $c>0, A=0$

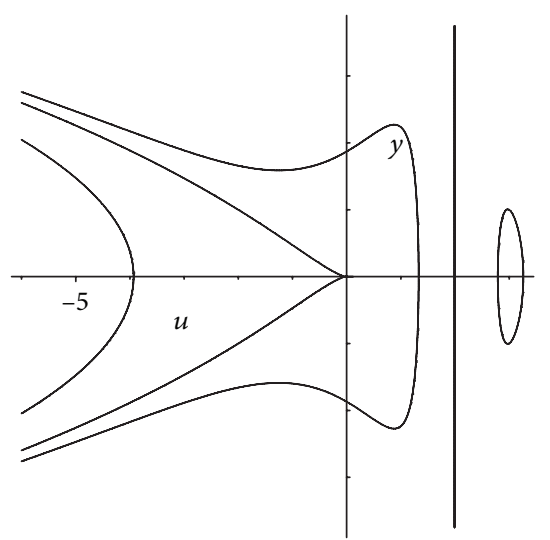

(m) $c>0, A=4 c$

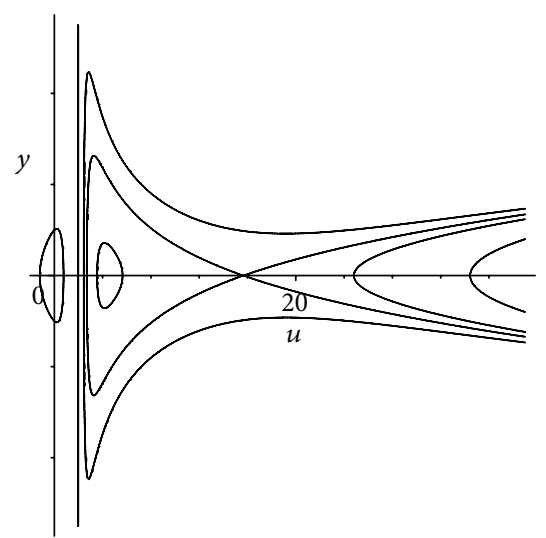

(j) $c>0,-(1 / 2) c<A<0$

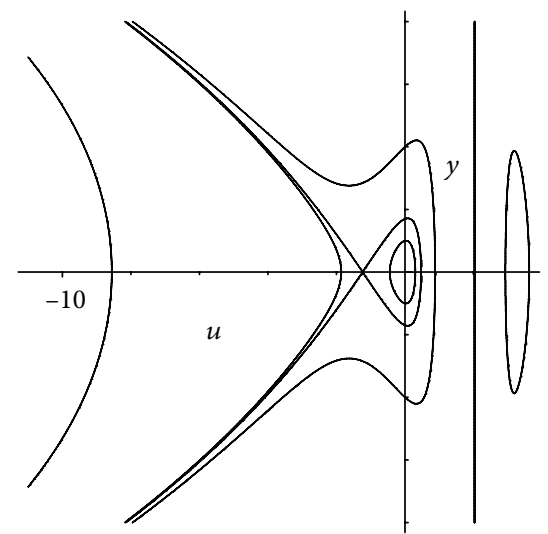

(l) $c>0,0<A<4 c$

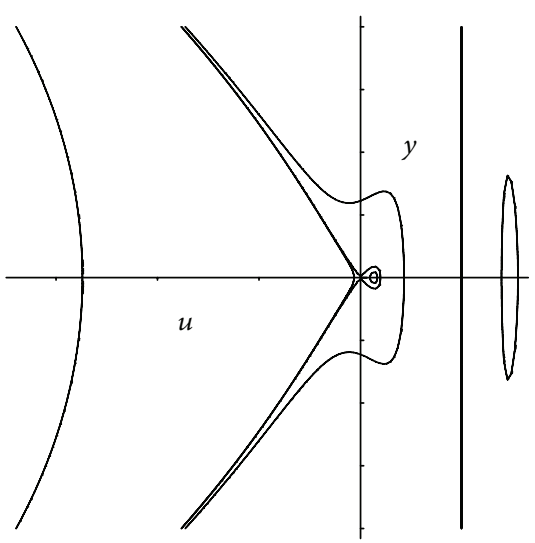

(n) $c>0, A>4 c$

FIGURE 1: Bifurcation sets and phase portraits of system (10).

\section{Exact Travelling Wave Solutions of System (4)}

Denote that

$$
\begin{aligned}
& h_{0}=4 c^{3}, \\
& h_{1}=\frac{c^{2}\left(c\left(7 A^{2}+2 A c+4 c^{2}\right)+\left(A^{2}+2 A c-4 c^{2}\right) \sqrt{\Delta}\right)}{A(c-\sqrt{\Delta})},
\end{aligned}
$$

$$
h_{2}=\frac{c^{2}\left(-c\left(7 A^{2}+2 A c+4 c^{2}\right)+\left(A^{2}+2 A c-4 c^{2}\right) \sqrt{\Delta}\right)}{A(-c-\sqrt{\Delta})}
$$

From Figure 1, we give some exact travelling wave solutions of system (4) as follows.

3.1. Different Kinds of Periodic Wave Solutions. From Figures $1(\mathrm{~d})$ and $1(\mathrm{k})$, we see that there is one periodic orbit 
of system $(10)$ defined by $H(u, y)=h$ if and only if one of the following conditions holds:

$$
\begin{aligned}
& \left(a_{1}\right) c<0, A=0, h<h_{0} ; \\
& \left(a_{2}\right) c>0, A=0, h>h_{0} .
\end{aligned}
$$

The periodic orbit passes points $\left(u_{m}, 0\right)$ and $\left(u_{M}, 0\right)$, where $u_{M, m}=\left(-h+4 c^{3} \pm \sqrt{h^{2}+8 c^{3} h-48 c^{6}}\right) / 8 c^{2}$. Its expression is

$$
y= \pm \frac{2 c \sqrt{\left(u_{M}-u\right)\left(u-u_{m}\right)}}{c-u}, \quad u_{m} \leq u \leq u_{M} .
$$

If introducing a new parametric variable $\chi$ and letting

$$
d \xi=(c-u) d \chi
$$

then we have

$$
\frac{d u}{d \chi}=(c-u) y
$$

Substituting (15) into (17) and integrating it along the periodic orbit yield the following:

$$
\int_{u}^{u_{M}} \frac{d s}{\sqrt{\left(u_{M}-s\right)\left(s-u_{m}\right)}}=2|c \chi| .
$$

Completing (18) and using (8) and (16), we can get a periodic wave solution of system (4) as follows:

$$
\begin{gathered}
u(x, t)=u_{m} \sin ^{2}(c \chi)+u_{M} \cos ^{2}(c \chi), \\
\rho(x, t)=\frac{2 c^{2}}{u_{m} \sin ^{2}(c \chi)+u_{M} \cos ^{2}(c \chi)-c} \\
t=\frac{1}{c}\left(x-\left(\left(c-\frac{1}{2}\left(u_{m}+u_{M}\right)\right) \chi+\frac{u_{m}-u_{M}}{4 c} \sin (2 c \chi)\right)\right) .
\end{gathered}
$$

From Figures 1(a), 1(b), 1(c), 1(h), 1(i), and 1(j), we see that there are one periodic orbit and an open curve of system (10) defined by $H(u, y)=h$ if and only if one of the following conditions holds:

$$
\begin{aligned}
& \left(b_{1}\right) c<0, A<4 c, h<h_{2} ; \\
& \left(b_{2}\right) c<0, A<4 c, h_{0}<h<h_{1} ; \\
& \left(b_{3}\right) c<0, A=4 c, h<h_{2} ; \\
& \left(b_{4}\right) c<0,4 c<A<0, h>h_{2} ; \\
& \left(b_{5}\right) c<0,4 c<A<0, h_{1}<h<h_{0} ; \\
& \left(b_{6}\right) c>0, \quad A<-(1 / 2) c, h>h_{0} ; \\
& \left(b_{7}\right) c>0, \quad A=-(1 / 2) c, h>h_{0} ; \\
& \left(b_{8}\right) c>0,-(1 / 2) c<A<0, h>h_{0} ; \\
& \left(b_{9}\right) c>0,-(1 / 2) c<A<0, h_{2}<h<h_{1} .
\end{aligned}
$$

The periodic orbit passing points $\left(\gamma_{3}, 0\right)$ and $\left(\gamma_{2}, 0\right)$ and the open curve passing point $\left(\gamma_{1}, 0\right)$, where $\gamma_{1}, \gamma_{2}, \gamma_{3}$ $\left(\gamma_{3}<\gamma_{2}<\gamma_{1}\right)$, are three real roots of $A z^{3}+c(4 c-A) z^{2}+(h-$ $\left.4 c^{3}\right) z+c\left(4 c^{3}-h\right)=0$. Their expressions are, respectively,

$$
\begin{gathered}
y= \pm \frac{\sqrt{-A\left(\gamma_{1}-u\right)\left(\gamma_{2}-u\right)\left(u-\gamma_{3}\right)}}{c-u}, \quad \gamma_{3} \leq u \leq \gamma_{2}, \\
y= \pm \frac{\sqrt{-A\left(u-\gamma_{1}\right)\left(u-\gamma_{2}\right)\left(u-\gamma_{3}\right)}}{c-u}, \quad u \geq \gamma_{1} .
\end{gathered}
$$

Substituting (20) into (17) and integrating it along the periodic orbit yield equation

$$
\int_{\gamma_{3}}^{u} \frac{d s}{\sqrt{\left(\gamma_{1}-s\right)\left(\gamma_{2}-s\right)\left(s-\gamma_{3}\right)}}=\sqrt{-A}|\chi| .
$$

Completing (22) and using (8) and (16), we can get a periodic wave solution of system (4) as follows:

$$
\begin{gathered}
u(x, t)=\gamma_{3}+\left(\gamma_{2}-\gamma_{3}\right) \operatorname{sn}^{2}(\omega \chi, k), \\
\rho(x, t)=\frac{2 c^{2}}{\left(\gamma_{3}-c\right)+\left(\gamma_{2}-\gamma_{3}\right) \operatorname{sn}^{2}(\omega \chi, k)}, \\
t=\frac{1}{c}\left(x-\left(\left(c-\gamma_{3}\right) \chi+\frac{\gamma_{3}-\gamma_{2}}{\omega k^{2}}\right.\right. \\
\quad \times(\omega \chi-\mathrm{E}(\operatorname{am}(\omega \chi, k), k)))),
\end{gathered}
$$

where $\omega=(1 / 2) \sqrt{-A\left(\gamma_{1}-\gamma_{3}\right)}, k=\sqrt{\left(\gamma_{2}-\gamma_{3}\right) /\left(\gamma_{1}-\gamma_{3}\right)}$, $\operatorname{sn}(\cdot, k)$ is the Jacobian elliptic function with the modulus $k$, $\mathrm{E}\left(\operatorname{am}\left(u_{1}, k\right), k\right)$ is the normal elliptic integral of the second kind, and am $\left(u_{1}, k\right)$ reads amplitude $u_{1}$ (see [18]).

Substituting (21) into (17) and integrating it along the open curve yield the following:

$$
\int_{u}^{+\infty} \frac{d s}{\sqrt{\left(s-\gamma_{1}\right)\left(s-\gamma_{2}\right)\left(s-\gamma_{3}\right)}}=\sqrt{-A}|\chi| .
$$

Completing (24) and using (8) and (16), we can get a periodic blow-up wave solution of system (4) as follows:

$$
\begin{gathered}
u(x, t)=\gamma_{3}+\left(\gamma_{1}-\gamma_{3}\right) \mathrm{ns}^{2}(\omega \chi, k), \\
\rho(x, t)=\frac{2 c^{2}}{\left(\gamma_{3}-c\right)+\left(\gamma_{1}-\gamma_{3}\right) \mathrm{ns}^{2}(\omega \chi, k)}, \\
t=\frac{1}{c}\left(x-\left(\left(c-\gamma_{1}\right) \chi+\frac{\gamma_{1}-\gamma_{3}}{\omega}\right.\right. \\
\times(\mathrm{E}(\operatorname{am}(\omega \chi, k), k)+\operatorname{dn}(\omega \chi, k) \operatorname{cs}(\omega \chi, k)))),
\end{gathered}
$$

where $\omega=(1 / 2) \sqrt{-A\left(\gamma_{1}-\gamma_{3}\right)}, k=\sqrt{\left(\gamma_{2}-\gamma_{3}\right) /\left(\gamma_{1}-\gamma_{3}\right)}$, $\mathrm{ns}(\cdot, k), \operatorname{dn}(\cdot, k)$, and $\operatorname{cs}(\cdot, k)$ are the Jacobian elliptic functions (see [18]).

From Figures 1(e), 1(f), 1(g), 1(l), 1(m), and 1(n), we see that there are one periodic orbit and an open curve of system 
(10) defined by $H(u, y)=h$ if and only if one of the following conditions holds:

$$
\begin{aligned}
& \left(c_{1}\right) c<0,0<A<-(1 / 2) c, h<h_{0} \\
& \left(c_{2}\right) c<0, \quad 0<A<-(1 / 2) c, h_{2}<h<h_{1} \\
& \left(c_{3}\right) c<0, A=-(1 / 2) c, h<h_{0} \\
& \left(c_{4}\right) c<0, A>-(1 / 2) c, h<h_{0} \\
& \left(c_{5}\right) c>0,0<A<4 c, h_{0}<h<h_{2} \\
& \left(c_{6}\right) c>0, \quad 0<A<4 c, h<h_{1} \\
& \left(c_{7}\right) c>0, A=4 c, h<h_{1} \\
& \left(c_{8}\right) c>0, A>4 c, h_{2}<h<h_{0} \\
& \left(c_{9}\right) c>0, A>4 c, h<h_{1} .
\end{aligned}
$$

The periodic orbit passes points $\left(\gamma_{1}, 0\right)$ and $\left(\gamma_{2}, 0\right)$, and the open curve passes point $\left(\gamma_{3}, 0\right)$, where $\gamma_{1}, \gamma_{2}, \gamma_{3}\left(\gamma_{3}<\gamma_{2}<\gamma_{1}\right)$ are three real roots of $A z^{3}+c(4 c-A) z^{2}+\left(h-4 c^{3}\right) z+c\left(4 c^{3}-h\right)=$ 0 . Their expressions are, respectively,

$$
\begin{array}{ll}
y= \pm \frac{\sqrt{A\left(\gamma_{1}-u\right)\left(u-\gamma_{2}\right)\left(u-\gamma_{3}\right)}}{c-u}, & \gamma_{2} \leq u \leq \gamma_{1}, \\
y= \pm \frac{\sqrt{A\left(\gamma_{1}-u\right)\left(\gamma_{2}-u\right)\left(\gamma_{3}-u\right)}}{c-u}, & u \leq \gamma_{3} .
\end{array}
$$

Substituting (26) into (17) and integrating it along the periodic orbit yield the following:

$$
\int_{u}^{\gamma_{1}} \frac{d s}{\sqrt{\left(\gamma_{1}-s\right)\left(s-\gamma_{2}\right)\left(s-\gamma_{3}\right)}}=\sqrt{A}|\chi| .
$$

Completing (28) and using (8) and (16), we can get a periodic wave solution of system (4) as follows:

$$
\begin{gathered}
u(x, t)=\gamma_{1}+\left(\gamma_{2}-\gamma_{1}\right) \operatorname{sn}^{2}(\omega \chi, k), \\
\rho(x, t)=\frac{2 c^{2}}{\left(\gamma_{1}-c\right)+\left(\gamma_{2}-\gamma_{1}\right) \operatorname{sn}^{2}(\omega \chi, k)}, \\
t=\frac{1}{c}\left(x-\left(\left(c-\gamma_{1}\right) \chi+\frac{\gamma_{1}-\gamma_{2}}{\omega k^{2}}\right.\right. \\
\quad \times(\omega \chi-\mathrm{E}(\operatorname{am}(\omega \chi, k), k)))),
\end{gathered}
$$

where $\omega=(1 / 2) \sqrt{A\left(\gamma_{1}-\gamma_{3}\right)}, k=\sqrt{\left(\gamma_{1}-\gamma_{2}\right) /\left(\gamma_{1}-\gamma_{3}\right)}$.

Substituting (27) into (17) and integrating it along the open curve yield the following:

$$
\int_{-\infty}^{u} \frac{d s}{\sqrt{\left(\gamma_{1}-s\right)\left(\gamma_{2}-s\right)\left(\gamma_{3}-s\right)}}=\sqrt{A}|\chi| .
$$

Completing (30) and using (8) and (16), we can get a periodic blow-up wave solution of system (4) as follows:

$$
\begin{gathered}
u(x, t)=\gamma_{1}+\left(\gamma_{3}-\gamma_{1}\right) \mathrm{ns}^{2}(\omega \chi, k), \\
\rho(x, t)=\frac{2 c^{2}}{\left(\gamma_{1}-c\right)+\left(\gamma_{3}-\gamma_{1}\right) \mathrm{ns}^{2}(\omega \chi, k)}, \\
t=\frac{1}{c}\left(x-\left(\left(c-\gamma_{3}\right) \chi+\frac{\gamma_{3}-\gamma_{1}}{\omega}\right.\right. \\
\times(\mathrm{E}(\operatorname{am}(\omega \chi, k), k)+\operatorname{dn}(\omega \chi, k) \mathrm{cs}(\omega \chi, k)))),
\end{gathered}
$$

where $\omega=(1 / 2) \sqrt{A\left(\gamma_{1}-\gamma_{3}\right)}, k=\sqrt{\left(\gamma_{1}-\gamma_{2}\right) /\left(\gamma_{1}-\gamma_{3}\right)}$.

3.2. Solitary Wave and Blow-Up Wave Solutions. From Figure 1(a), we see that there are one homoclinic orbit and an open curve of system (10) defined by $H(\phi, y)=h_{0}$ when $c<0, A<4 c$. The homoclinic orbit connecting with saddle point $(0,0)$ and passes point $\left(u_{m}, 0\right)$, and the open curve passes saddle point $(0,0)$, where $u_{m}=c(A-4 c) / A$. Their expressions are, respectively,

$$
\begin{array}{ll}
y= \pm \frac{u \sqrt{-A\left(u-u_{m}\right)}}{c-u}, & u_{m} \leq u<0, \\
y= \pm \frac{u \sqrt{-A\left(u-u_{m}\right)}}{c-u}, & u \geq 0 .
\end{array}
$$

Substituting (32) into (17) and integrating it along the homoclinic orbit yield the following:

$$
\int_{u_{m}}^{u} \frac{d s}{s \sqrt{s-u_{m}}}=-\sqrt{-A}|\chi| .
$$

Completing (34) and using (8) and (16), we can get a solitary wave solution of system (4) as follows:

$$
\begin{gathered}
u(x, t)=\frac{c(A-4 c)}{A} \operatorname{sech}^{2}(\omega \chi), \\
\rho(x, t)=\frac{2 A c}{(A-4 c) \operatorname{sech}^{2}(\omega \chi)-A}, \\
t=\frac{1}{c}\left(x-c\left(\chi-\frac{(A-4 c)}{\omega A} \tanh (\omega \chi)\right)\right),
\end{gathered}
$$

where $\omega=(1 / 2) \sqrt{c(A-4 c)}$.

Substituting (33) into (17) and integrating it along the open curve yield the following:

$$
\int_{u}^{+\infty} \frac{d s}{s \sqrt{s-u_{m}}}=\sqrt{-A}|\chi| .
$$


Completing (36) and using (8) and (16), we can get a blow-up wave solution of system (4) as follows:

$$
\begin{gathered}
u(x, t)=\frac{c(4 c-A)}{A} \operatorname{csch}^{2}(\omega \chi), \\
\rho(x, t)=\frac{2 A c}{(4 c-A) \operatorname{csch}^{2}(\omega \chi)-A}, \\
t=\frac{1}{c}\left(x-c\left(\chi+\frac{(A-4 c)}{\omega A} \operatorname{coth}(\omega \chi)\right)\right),
\end{gathered}
$$

where $\omega=(1 / 2) \sqrt{c(A-4 c)}$.

From Figure 1(n), we see that there are one homoclinic orbit and an open curve of system (10) defined by $H(\phi, y)=$ $h_{0}$ when $c>0, A>4 c$. The homoclinic orbit connects with saddle point $(0,0)$ and passes point $\left(u_{M}, 0\right)$, and the open curve passes saddle point $(0,0)$, where $u_{M}=c(A-4 c) / A$. Their expressions are, respectively,

$$
\begin{array}{ll}
y= \pm \frac{u \sqrt{A\left(u_{M}-u\right)}}{c-u}, & 0<u \leq u_{M} \\
y= \pm \frac{u \sqrt{A\left(u_{M}-u\right)}}{c-u}, & u \leq 0 .
\end{array}
$$

Substituting (38) into (17) and integrating it along the homoclinic orbit yield the following:

$$
\int_{u}^{u_{M}} \frac{d s}{s \sqrt{u_{M}-s}}=\sqrt{A}|\chi| .
$$

Completing (40) and using (8) and (16), we can get a solitary wave solution of system (4) the same as (35).

Substituting (39) into (17) and integrating it along the open curve yield the following:

$$
\int_{-\infty}^{u} \frac{d s}{s \sqrt{u_{M}-s}}=\sqrt{A}|\chi| .
$$

Completing (41) and using (8) and (16), we can get a blow-up wave solution of system (4) same as (37).

From Figure 1(b), we see that there is an open curve of system (10) defined by $H(\phi, y)=h_{0}$ passing cusp $(0,0)$ when $c<0, A=4 c$. Its expression is

$$
y= \pm \frac{2 u \sqrt{-c u}}{c-u}, \quad u \geq 0
$$

Substituting (42) into (17) and integrating it along the open curve yield the following:

$$
\int_{u}^{+\infty} \frac{d s}{s \sqrt{-c s}}=2|\chi| .
$$

Completing (43) and using (8) and (16), we can get a blow-up wave solution of system (4) as follows:

$$
\begin{gathered}
u(x, t)=-\frac{1}{c \chi^{2}}, \\
\rho(x, t)=-\frac{2 c^{3} \chi^{2}}{1+c^{2} \chi^{2}}, \\
t=\frac{1}{c}\left(x-\left(c \chi-\frac{1}{c \chi}\right)\right) .
\end{gathered}
$$

From Figure $1(\mathrm{~m})$, we see that there is an open curve of system (10) defined by $H(\phi, y)=h_{0}$ passes cusp $(0,0)$ when $c>0, A=4 c$. Its expression is

$$
y= \pm \frac{2 u \sqrt{-c u}}{c-u}, \quad u \leq 0 .
$$

Substituting (45) into (17) and integrating it along the open curve yield the following:

$$
\int_{-\infty}^{u} \frac{d s}{s \sqrt{-c s}}=-2|\chi| .
$$

Completing (46) and using (8) and (16), we can get a blow-up wave solution of system (4) the same as (44).

From Figure 1(c), we see that there are one homoclinic orbit and an open curve of system (10) defined by $H(\phi, y)=$ $h_{1}$ when $c<0,4 c<A<0$. The homoclinic orbit connects with saddle point $\left(u_{1}, 0\right)$ and passes point $\left(u_{m}, 0\right)$, and the open curve passes saddle point $\left(u_{1}, 0\right)$, where $u_{m}=-c((A+$ $2 c)+2 \sqrt{c(2 A+c)}) / A$. Their expressions are, respectively,

$$
\begin{array}{ll}
y= \pm \frac{\left(u_{1}-u\right) \sqrt{-A\left(u-u_{m}\right)}}{c-u}, & u_{m} \leq u<u_{1}, \\
y= \pm \frac{\left(u-u_{1}\right) \sqrt{-A\left(u-u_{m}\right)}}{c-u}, & u \geq u_{1} .
\end{array}
$$

Substituting (47) into (17) and integrating it along the homoclinic orbit yield the following:

$$
\int_{u_{m}}^{u} \frac{d s}{\left(u_{1}-s\right) \sqrt{s-u_{m}}}=\sqrt{-A}|\chi| .
$$

Completing (49) and using (8) and (16), we can get a solitary wave solution of system (4) as follows:

$$
\begin{gathered}
u(x, t)=\Omega+\left(u_{1}-\Omega\right) \tanh ^{2}(\omega \chi), \\
\rho(x, t)=\frac{2 c^{2}}{(\Omega-c)+\left(u_{1}-\Omega\right) \tanh ^{2}(\omega \chi)}, \\
t=\frac{1}{c}\left(x-\left(\left(c-u_{1}\right) \chi+\frac{u_{1}-\Omega}{\omega} \tanh (\omega \chi)\right)\right),
\end{gathered}
$$

where $\Omega=-c((A+2 c)+2 \sqrt{c(2 A+c)}) / A, \omega=(1 / 2)$ $\sqrt{A\left(\Omega-u_{1}\right)}$. 
Substituting (48) into (17) and integrating it along the open curve yield the following:

$$
\int_{u}^{+\infty} \frac{d s}{\left(s-u_{1}\right) \sqrt{s-u_{m}}}=\sqrt{-A}|\chi| .
$$

Completing (51) and using (8) and (16), we can get a blow-up wave solution of system (4) as follows:

$$
\begin{gathered}
u(x, t)=\Omega+\left(u_{1}-\Omega\right) \operatorname{coth}^{2}(\omega \chi), \\
\rho(x, t)=\frac{2 c^{2}}{(\Omega-c)+\left(u_{1}-\Omega\right) \operatorname{coth}^{2}(\omega \chi)}, \\
t=\frac{1}{c}\left(x-\left(\left(c-u_{1}\right) \chi+\frac{u_{1}-\Omega}{\omega} \operatorname{coth}(\omega \chi)\right)\right),
\end{gathered}
$$

where $\Omega=-c((A+2 c)+2 \sqrt{c(2 A+c)}) / A, \omega=(1 / 2)$ $\sqrt{A\left(\Omega-u_{1}\right)}$.

From Figure 1(e), we see that there are one homoclinic orbit and an open curve of system (10) defined by $H(\phi, y)=$ $h_{1}$ when $c<0,0<A<-(1 / 2) c$. The homoclinic orbit connects with saddle point $\left(u_{1}, 0\right)$ and passes point $\left(u_{M}, 0\right)$, and the open curve passes saddle point $\left(u_{1}, 0\right)$, where $u_{M}=-c((A+2 c)+2 \sqrt{c(2 A+c)}) / A$. Their expressions are, respectively,

$$
\begin{array}{ll}
y= \pm \frac{\left(u-u_{1}\right) \sqrt{A\left(u_{M}-u\right)}}{c-u}, & u_{1}<u \leq u_{M} \\
y= \pm \frac{\left(u_{1}-u\right) \sqrt{A\left(u_{M}-u\right)}}{c-u}, & u \leq u_{1} .
\end{array}
$$

Substituting (53) into (17) and integrating it along the homoclinic orbit yield the following:

$$
\int_{u}^{u_{M}} \frac{d s}{\left(s-u_{1}\right) \sqrt{u_{M}-s}}=\sqrt{A}|\chi| .
$$

Completing (55) and using (8) and (16), we can get a solitary wave solution of system (4) the same as (50).

Substituting (54) into (17) and integrating it along the open curve yield the following:

$$
\int_{-\infty}^{u} \frac{d s}{\left(u_{1}-s\right) \sqrt{u_{M}-s}}=\sqrt{A}|\chi| .
$$

Completing (56) and using (8) and (16), we can get a blow-up wave solution of system (4) the same as (52).

From Figure 1(f), we see that there is an open curve of system (10) defined by $H(\phi, y)=-(19 / 2) c^{3}$ passes cusp $(3 c, 0)$ when $c<0, A=-(1 / 2) c$. Its expression is

$$
y= \pm \frac{(3 c-u) \sqrt{-(1 / 2) c(3 c-u)}}{c-u}, \quad u \leq 3 c .
$$

Substituting (57) into (17) and integrating it along the open curve yield the following:

$$
\int_{-\infty}^{u} \frac{d s}{(3 c-s) \sqrt{3 c-s}}=\sqrt{-\frac{c}{2}}|\chi| .
$$

Completing (58) and using (8) and (16), we can get a blow-up wave solution of system (4) as follows:

$$
\begin{gathered}
u(x, t)=3 c+\frac{8}{c \chi^{2}}, \\
\rho(x, t)=\frac{2 c^{3} \chi^{2}}{8+2 c^{2} \chi^{2}}, \\
t=\frac{1}{c}\left(x+\left(2 c \chi-\frac{8}{c \chi}\right)\right) .
\end{gathered}
$$

From Figure 1(i), we see that there is an open curve of system $(10)$ defined by $H(\phi, y)=-(19 / 2) c^{3}$ passes cusp $(3 c, 0)$ when $c>0, A=-(1 / 2) c$. Its expression is

$$
y= \pm \frac{(u-3 c) \sqrt{(1 / 2) c(u-3 c)}}{c-u}, \quad 3 c \leq u .
$$

Substituting (60) into (17) and integrating it along the open curve yield the following:

$$
\int_{u}^{+\infty} \frac{d s}{(s-3 c) \sqrt{s-3 c}}=\sqrt{\frac{\bar{c}}{2}}|\chi| .
$$

Completing (61) and using (8) and (16), we can get a blow-up wave solution of system (4) the same as (59).

From Figure 1(j), we see that there are one homoclinic orbit and an open curve of system (10) defined by $H(\phi, y)=$ $h_{2}$ when $c>0,-(1 / 2) c<A<0$. The homoclinic orbit connects with saddle point $\left(u_{2}, 0\right)$ and passes point $\left(u_{m}, 0\right)$, and the open curve passes saddle point $\left(u_{2}, 0\right)$, where $u_{m}=-c((A+2 c)-2 \sqrt{c(2 A+c)}) / A$. Their expressions are, respectively,

$$
\begin{array}{ll}
y= \pm \frac{\left(u_{2}-u\right) \sqrt{-A\left(u-u_{m}\right)}}{c-u}, & u_{m} \leq u<u_{2}, \\
y= \pm \frac{\left(u-u_{2}\right) \sqrt{-A\left(u-u_{m}\right)}}{c-u}, & u \geq u_{2} .
\end{array}
$$

Substituting (62) into (17) and integrating it along the homoclinic orbit yield the following:

$$
\int_{u_{m}}^{u} \frac{d s}{\left(u_{2}-s\right) \sqrt{s-u_{m}}}=\sqrt{-A}|\chi| .
$$

Completing (64) and using (8) and (16), we can get a solitary wave solution of system (4) as follows:

$$
\begin{gathered}
u(x, t)=\Omega+\left(u_{2}-\Omega\right) \tanh ^{2}(\omega \chi), \\
\rho(x, t)=\frac{2 c^{2}}{(\Omega-c)+\left(u_{2}-\Omega\right) \tanh ^{2}(\omega \chi)}, \\
t=\frac{1}{c}\left(x-\left(\left(c-u_{2}\right) \chi+\frac{u_{2}-\Omega}{\omega} \tanh (\omega \chi)\right)\right),
\end{gathered}
$$

where $\Omega=-c((A+2 c)-2 \sqrt{c(2 A+c)}) / A, \omega=(1 / 2)$ $\sqrt{A\left(\Omega-u_{2}\right)}$. 
Substituting (63) into (17) and integrating it along the open curve yield the following:

$$
\int_{u}^{+\infty} \frac{d s}{\left(s-u_{2}\right) \sqrt{s-u_{m}}}=\sqrt{-A}|\chi| .
$$

Completing (66) and using (8) and (16), we can get a blow-up wave solution of system (4) as follows:

$$
\begin{gathered}
u(x, t)=\Omega+\left(u_{2}-\Omega\right) \operatorname{coth}^{2}(\omega \chi), \\
\rho(x, t)=\frac{2 c^{2}}{(\Omega-c)+\left(u_{2}-\Omega\right) \operatorname{coth}^{2}(\omega \chi)}, \\
t=\frac{1}{c}\left(x-\left(\left(c-u_{2}\right) \chi+\frac{u_{2}-\Omega}{\omega} \operatorname{coth}(\omega \chi)\right)\right),
\end{gathered}
$$

where $\Omega=-c((A+2 c)-2 \sqrt{c(2 A+c)}) / A, \omega=$ $(1 / 2) \sqrt{A\left(\Omega-u_{2}\right)}$.

From Figure 1(l), we see that there are one homoclinic orbit and an open curve of system (10) defined by $H(\phi, y)=$ $h_{2}$ when $c>0,0<A<4 c$. The homoclinic orbit connects with saddle point $\left(u_{2}, 0\right)$ and passes point $\left(u_{M}, 0\right)$, and the open curve passes saddle point $\left(u_{2}, 0\right)$, where $u_{M}=-c((A+$ $2 c)-2 \sqrt{c(2 A+c)}) / A$. Their expressions are, respectively,

$$
\begin{array}{ll}
y= \pm \frac{\left(u-u_{2}\right) \sqrt{A\left(u_{M}-u\right)}}{c-u}, & u_{2}<u \leq u_{M} \\
y= \pm \frac{\left(u_{2}-u\right) \sqrt{A\left(u_{M}-u\right)}}{c-u}, & u \leq u_{2} .
\end{array}
$$

Substituting (68) into (17) and integrating it along the homoclinic orbit yield the following:

$$
\int_{u}^{u_{M}} \frac{d s}{\left(s-u_{2}\right) \sqrt{u_{M}-s}}=\sqrt{A}|\chi| .
$$

Completing (70) and using (8) and (16), we can get a solitary wave solution of system (4) the same as (65).

Substituting (69) into (17) and integrating it along the open curve yield the following:

$$
\int_{-\infty}^{u} \frac{d s}{\left(u_{2}-s\right) \sqrt{u_{M}-s}}=\sqrt{A}|\chi| .
$$

Completing (71) and using (8) and (16), we can get a blow-up wave solution of system (4) the same as (67).

\section{Conclusion}

In this paper, we studied the bifurcations of travelling wave solutions of a generalized two-component Hunter-Saxton system and obtained different kinds of periodic wave solutions, which concluded periodic blow-up wave and periodic loop solutions and so forth. Some solitary wave and blowup wave solutions are also obtained. The results of this paper have enriched the results of [10-12].

\section{Conflict of Interests}

The authors declare that there is no conflict of interests regarding the publication of this paper.

\section{Acknowledgments}

The authors thank the referees very much for their perceptive comments and suggestions. This work is supported by the Natural Science Foundation of Yunnan Province, China (no. 2013FZ117), and the National Natural Science Foundation of China (no. 11161020).

\section{References}

[1] J. K. Hunter and R. Saxton, "Dynamics of director fields," SIAM Journal on Applied Mathematics, vol. 51, no. 6, pp. 1498-1521, 1991.

[2] B. Khesin and G. Misiołek, "Euler equations on homogeneous spaces and Virasoro orbits," Advances in Mathematics, vol. 176, no. 1, pp. 116-144, 2003.

[3] D. D. Holm, "Peakons," in Encyclopedia of Mathematical Physics, vol. 4, pp. 12-20, Academic Press, Oxford, UK, 2006.

[4] J. K. Hunter and Y. Zheng, "On a completely integrable nonlinear hyperbolic variational equation," Physica D: Nonlinear Phenomena, vol. 79, no. 2-4, pp. 361-386, 1994.

[5] M. Wunsch, "On the Hunter-Saxton system," Discrete and Continuous Dynamical Systems B, vol. 12, no. 3, pp. 647-656, 2009.

[6] A. V. Gurevich and K. P. Zybin, "Nondissipative gravitational turbulence," Soviet Physics-JETP, vol. 67, pp. 1-12, 1988.

[7] M. V. Pavlov, “The Gurevich-Zybin system," Journal of Physics A: Mathematical and General, vol. 38, no. 17, pp. 3823-3840, 2005.

[8] M. Wunsch, "The generalized Hunter-Saxton system," SIAM Journal on Mathematical Analysis, vol. 42, no. 3, pp. 1286-1304, 2010.

[9] D. Zuo, "A two-component $\mu$-Hunter-Saxton equation," Inverse Problems, vol. 26, no. 8, Article ID 085003, 2010.

[10] B. Moon and Y. Liu, "Wave breaking and global existence for the generalized periodic two-component Hunter-Saxton system," Journal of Differential Equations, vol. 253, no. 1, pp. 319-355, 2012.

[11] B. Moon, "Solitary wave solutions of the generalized twocomponent Hunter-Saxton system," Nonlinear Analysis, vol. 89, pp. 242-249, 2013.

[12] J. Zhang and L. Tian, "Wave-breaking criterion for the generalized weakly dissipative periodic two-component HunterSaxton system," Journal of Applied Mathematics, vol. 2013, Article ID 809824, 10 pages, 2013.

[13] J. Li and H. Dai, On the Study of Singular Nonlinear Traveling Wave Equation: Dynamical System Approach, Science Press, Bejing, China, 2007.

[14] Z. Liu and B. Guo, "Periodic blow-up solutions and their limit forms for the generalized Camassa-Holm equation," Progress in Natural Science, vol. 18, no. 3, pp. 259-266, 2008.

[15] B. He, Q. Meng, and Y. Long, "The bifurcation and exact peakons, solitary and periodic wave solutions for the Kudryashov-Sinelshchikov equation," Communications in Nonlinear Science and Numerical Simulation, vol. 17, no. 11, pp. 4137-4148, 2012. 
[16] B. He, "Bifurcations and exact bounded travelling wave solutions for a partial differential equation," Nonlinear Analysis: Real World Applications, vol. 11, no. 1, pp. 364-371, 2010.

[17] J. Li and Z. Liu, "Smooth and non-smooth traveling waves in a nonlinearly dispersive equation," Applied Mathematical Modelling, vol. 25, pp. 41-56, 2000.

[18] P. F. Byrd and M. D. Friedman, Handbook of Elliptic Integrals for Engineers and Scientists, Springer, Berlin, Germany, 1971. 


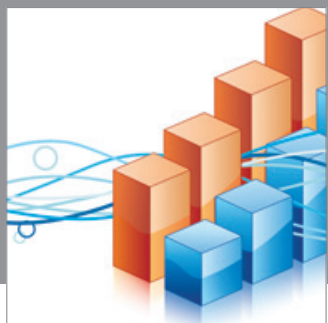

Advances in

Operations Research

mansans

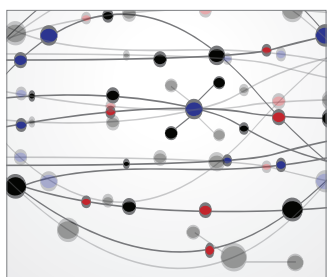

The Scientific World Journal
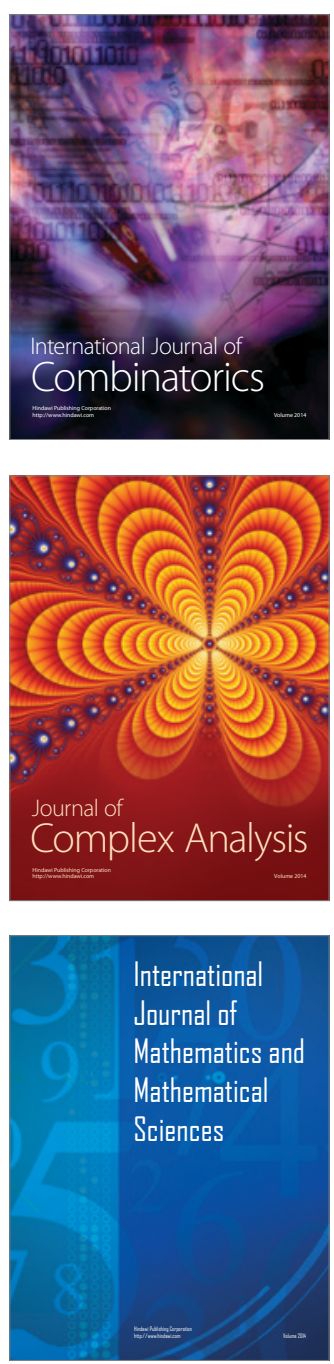
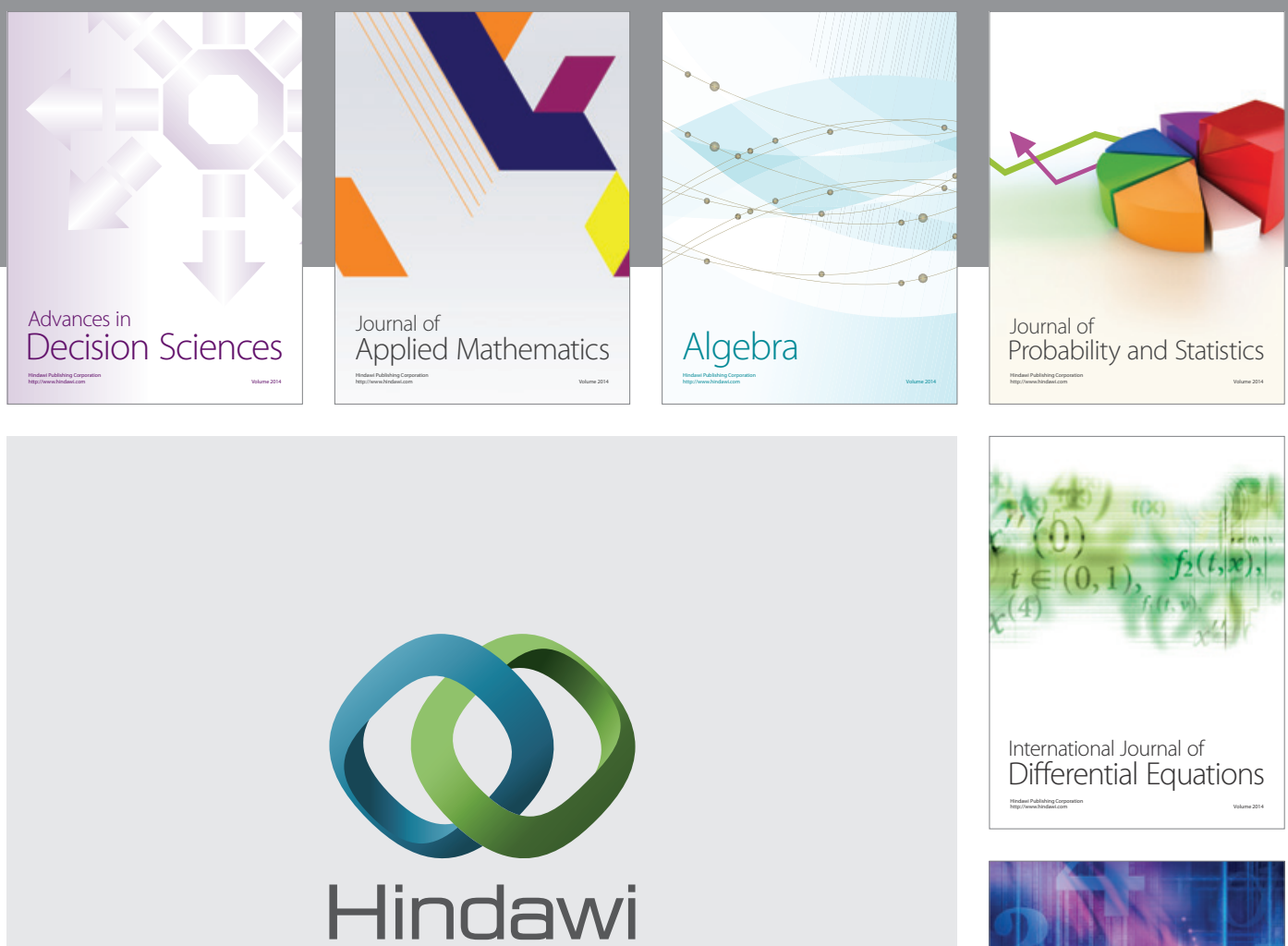

Submit your manuscripts at http://www.hindawi.com
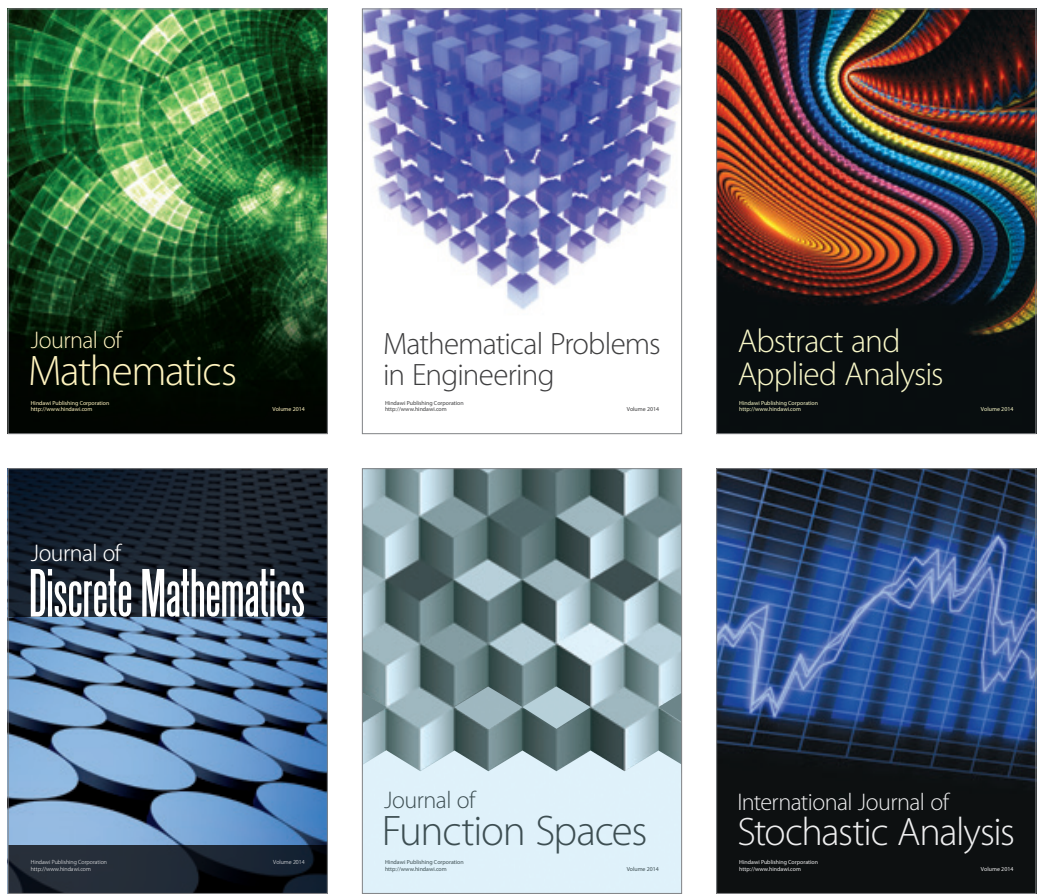

Journal of

Function Spaces

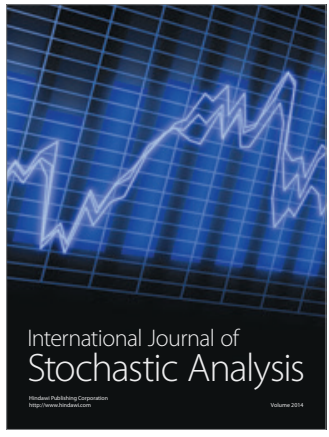

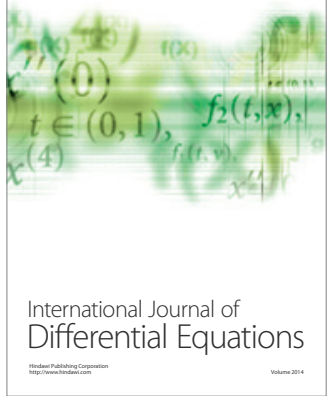
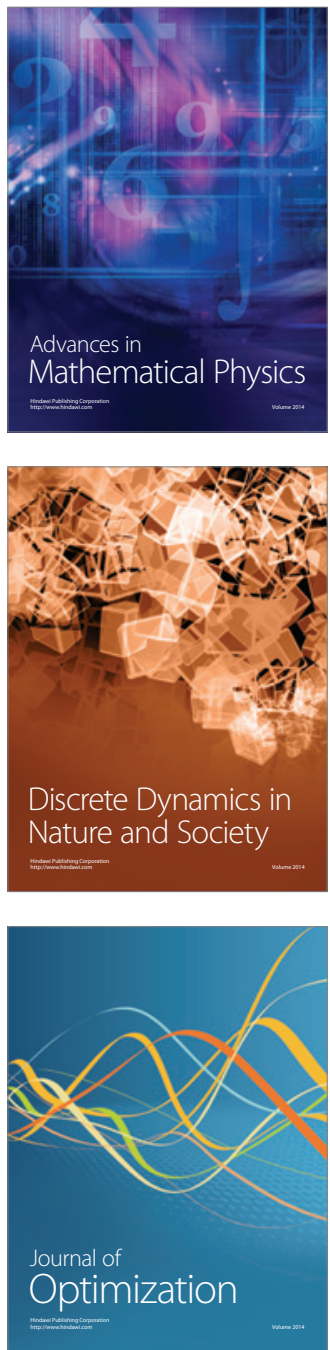